\title{
OPEN Peptide-HLA-based immunotherapeutics platforms for direct modulation of antigen-specific $T$ cells
}

\author{
Ronald D. Seidel ${ }^{1 凶}$, Zohra Merazga ${ }^{1}$, Dharma Raj Thapa ${ }^{1}$, Jonathan Soriano ${ }^{1}$, \\ Emily Spaulding ${ }^{1}$, Ahmet S. Vakkasoglu ${ }^{1}$, Paige Ruthardt ${ }^{1}$, Wynona Bautista ${ }^{1}$, \\ Steven N. Quayle ${ }^{1}$, Peter A. Kiener ${ }^{2}$, Simon Low ${ }^{1}$, John F. Ross ${ }^{1}$, Saso Cemerski ${ }^{1}$, Anish Suri ${ }^{1}$, \\ Steven C. Almo ${ }^{3}$ \& Rodolfo J. Chaparro ${ }^{1}$
}

Targeted pharmacologic activation of antigen-specific (AgS) T cells may bypass limitations inherent in current $\mathrm{T}$ cell-based cancer therapies. We describe two immunotherapeutics platforms for selective delivery of costimulatory ligands and peptide-HLA (pHLA) to AgS T cells. We engineered and deployed on these platforms an affinity-attenuated variant of interleukin-2, which selectively expands oligoclonal and polyfunctional AgS T cells in vitro and synergizes with CD80 signals for superior proliferation versus peptide stimulation.

There remains a significant need for immunotherapeutics to drive clinically effective anti-tumor T cell responses. Adoptive T cell therapies (ACT) have demonstrated impressive clinical results for several cancers using patientderived T cells activated ex vivo with potent T cell receptor (TCR), costimulatory and cytokine stimulation ${ }^{1}$. However, cellular therapies reach few patients due to complex manufacturing and reinfusion requirements, patient conditioning regimens and safety considerations. In contrast, systemic costimulation represents a scalable pharmacologic approach to cancer immunotherapy which has the intent of activating and expanding tumorspecific T cells directly within the patient. However, attempts to induce anti-tumor T cell responses using systemic agonism with agents such as anti-CD137 antibodies and high dose interleukin-2 (IL-2) are associated with significant risk of toxicity ${ }^{2,3}$. IL-2 is of particular interest as a potent cytokine capable of inducing the proliferation and differentiation of CD8 effector T cells (Teff), as well as other T, B, and NK lineages with anti-tumor potential. In the contexts of metastatic renal cell carcinoma and malignant melanoma, high-dose IL-2 can induce curative remissions in a minority of patients, associated with elevated levels of AgS Teff, but is dose limited by severe and potentially life-threatening toxicities such as vascular leak syndrome and cytokine release syndrome ${ }^{4}$. The anti-tumor effects of IL-2 are also indirectly limited by regulatory T cells (Treg), which expand efficiently to IL-2 in vivo due to high level expression of the high affinity IL-2 receptor. Elevated Treg counts, which can limit Teff responses, are associated with poor prognosis in cancer patients ${ }^{5}$. In addition, cancer immunotherapeutic combinations targeting distinct costimulatory pathways hold the potential of greatly amplifying T cell responses ${ }^{6}$. However, as illustrated by the combined inhibition of CTLA- 4 and PD-1 pathways in the treatment of metastatic melanoma, toxicities may also compound, limiting their utility ${ }^{7}$. Broader therapeutic use of IL-2 and other costimulatory axes in cancer will likely require focusing their effects on those $\mathrm{T}$ cells which stand to deliver the greatest therapeutic effect, in particular cancer AgS T cells.

Cancer vaccines represent another scalable pharmacologic approach to cancer immunotherapy. However, vaccine efficacy depends on antigen-presenting cell (APC) function, including trafficking, antigen-processing, costimulation versus co-inhibition, and susceptibility to tumor immunosuppression ${ }^{8}$. For example, dendritic cells are APC which play critical roles in the priming and maintenance of anti-tumor CD8 T cell responses via delivery of peptide-HLA and potent costimulatory ligands such as CD80, CD86, and CD137L ${ }^{8}$. However, dendritic cells are also replete with inhibitory ligands such as TIM-3, PD-L1, PD-L2, HVEM, B7-H3, B7-H4, IL-T3, and IL-4, which blunt $\mathrm{T}$ cell responses and are subject to local and distal tumor influence ${ }^{9}$. Therefore, safe, scalable and APC-independent immunotherapeutics are needed that will enable clinically effective levels of anti-tumor T cell activation and associated tumor cell killing. We have identified a potential solution to this challenge based

${ }^{1}$ Cue Biopharma, Cambridge, MA, USA. ${ }^{2}$ BioKien LLC, Harpswell, ME, USA. ${ }^{3}$ Albert Einstein College of Medicine, Bronx, NY, USA. ${ }^{\boxplus}$ email: seidel.ron@gmail.com 
on the natural signals governing T cell function: peptide-HLA and costimulatory ligands, embodied in the Immuno-STAT (Selective Targeting and Alteration of T cells) and Neo-STAT immunotherapeutics platforms. Immuno-STAT and Neo-STAT utilize compact, Fc-based architectures amenable to clinical manufacturing, and are designed to focus optimized signals for potent costimulatory axes such as IL-2 directly to AgS T cells in vivo, thereby enhancing anti-tumor $\mathrm{T}$ cell responses while avoiding indiscriminate immune activation.

\section{Results}

The Immuno-STAT framework comprises a covalent fusion of peptide epitope, MHC class I allele, co-modulator, and $\mathrm{Fc}$, which imparts avidity and symmetrical multivalency, sufficient for cognate $\mathrm{T}$ cell activation (Fig. 1a) ${ }^{10}$. Potential co-modulators, costimulatory and co-inhibitory ligands, may be fused to the $\mathrm{N}$ - or $\mathrm{C}$-terminus of the MHC-Fc heavy chain or the C-terminus of $\beta 2 \mathrm{~m}$, allowing organizational, compositional and stoichiometric flexibility. Our initial exploration of the Immuno-STAT platform utilized IL-2 as the co-modulator to activate and expand AgS cytotoxic effector T cells (Teff).

To identify an optimized IL-2-based Immuno-STAT framework, we evaluated a panel of constructs for relative potency, AgS selectivity, and manufacturability. Constructs comprised LCMV gp $\mathrm{g}_{33-41} / \mathrm{H}-2 \mathrm{D}^{\mathrm{b}}$, recognized by the murine TCR P14, fused at its N-terminus to variants of human IL-2 and C-terminally to an effectorattenuated murine IgG2a Fc. IL-2-attenuating mutations were included to limit IL-2Ra-dependent toxicity and Treg engagement as well as to reduce IL-2 affinity in favor of pMHC selectivity (see Supplementary Fig. 1) ${ }^{11,12}$. IL-2 stoichiometries were limited to two or four based on manufacturability which showed a significant drop in protein titer beyond four copies of IL-2 (see Supplementary Fig. 2). Human IL-2 exhibits potent activity on both human and mouse cells including phosphorylation of STAT5, an IL-2 receptor (IL-2R) proximal signaling molecule and phosphorylated STAT5 (pSTAT5) serves as an index of IL-2R engagement which correlates well with downstream consequences of IL-2R agonism such as proliferation and phenotypic marker expression ${ }^{12}$. We compared pSTAT5 induction for purified CD8 splenocytes from AgS P14 TCR transgenic mice to non$\mathrm{AgS} \mathrm{C57BL/6} \mathrm{mice} \mathrm{and} \mathrm{ranked} \mathrm{constructs} \mathrm{based} \mathrm{on} \operatorname{logEC} 50_{\mathrm{P} 14}$ (potency index), P14 minus C57BL/6 signal at $\mathrm{EC} 0_{\mathrm{P} 14}$ (selectivity index), and protein expression titer (see Supplementary Fig. 3 and Supplementary Table 1). Construct LCMV-IST-IL2. $\mathrm{FH}_{4}$ ranked highest followed by LCMV-IST-IL2.F or IL-2 ${ }^{\mathrm{F} 42 \mathrm{~A}}$, respectively. Dose responses for top candidates LCMV-IST-IL2.FH $\mathrm{FH}_{4}$ and LCMV-IST-IL2.F from this $_{4}$ initial screen were reevaluated over a broader concentration range and with greater resolution and compared with reference constructs bearing two or four copies of wildtype IL-2, LCMV-IST-IL2 2 or LCMV-IST-IL2 4 , respectively (Fig. 1b and see Supplementary Fig. 4). AgS potency, as indicated by the logarithm of the pSTAT5 EC50 for responding P14 splenocytes $\left(\operatorname{logEC} 50_{\mathrm{P} 14}\right.$ ), was not significantly different between LCMV-IST-IL2.

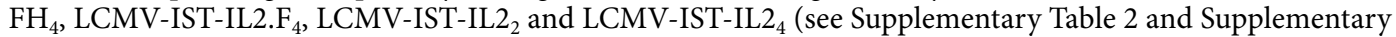
Fig. 5). Likewise, non-AgS potency $\left(\operatorname{logEC} 50_{\mathrm{B} 6}\right.$ ) was also similar for these constructs (see Supplementary Table 2 and Supplementary Fig. 5). AgS selectivity was measured first by the difference in normalized pSTAT5 signal at $\mathrm{EC} 50_{\mathrm{P} 14}$ for AgS (P14) splenocytes (i.e. 50\%) relative to non-AgS (C57BL/6) splenocytes as well as by the difference in the logEC50 for AgS versus non-AgS splenocytes (see Supplementary Fig. 6). Both measures of AgS selectivity were not significantly different across constructs LCMV-IST-IL2.FH ${ }_{4}$, LCMV-IST-IL2.F $_{4}$, LCMV-ISTIL $2_{2}$ and LCMV-IST-IL2 4 . Thus, significant F42A- and H16A-mediated decreases in IL-2R signaling potency or selectivity were not observed relative to reference constructs bearing two or four copies of wildtype IL-2 (LCMVIST-IL2 $2_{2}$ or LCMV-IST-IL2 $2_{4}$ ), presumably masked in part by the increased IL-2 stoichiometry.

We next generated and tested humanized Immuno-STATs bearing IL2. $\mathrm{FH}_{4}$, effector-attenuated human IgG1, HLA-A ${ }^{\star} 0201$ and model epitopes CMV pp65 ${ }_{495-503}$ or MART1 $1_{26-35}$ (Fig. 1c and see Supplementary Fig. 7). For multiple donors, we determined the frequency of dual-tetramer-positive AgS CD8 T cells following a 10 day incubation of human PBMC with specific IST-IL2.FH (Fig. $1 \mathrm{~d}$ and see Supplemental Fig. 8). CMV-IST-IL2.FH $_{4}$, but not CMV-IST (no IL-2 fusion) was able to expand CMV-specific CD8 T cells from PBMC, indicating that proliferation was dependent on the presence of IL2. $\mathrm{FH}_{4}$ (see Supplementary Fig. 9). Similarly, CMV-IST in the presence, but not absence, of recombinant human IL-2 (wildtype)-expanded CMV-specific CD8 T cells in vitro, further supporting the requirement for IL-2R agonism for Immuno-STAT activity (data not shown). Peak expansions (> 30 fold) were similar for PBMC CD8 T cells responsive to CMV-IST-IL2.FH or MART-IST-IL2.FH $_{4}$

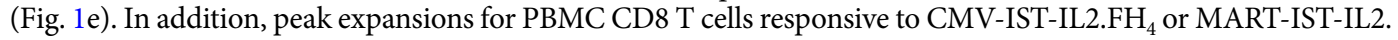
$\mathrm{FH}_{4}$ were achieved at similar Immuno-STAT concentrations (see Supplementary Fig. 10). However, higher CMVversus MART-specific frequencies were present in expanded and media cultures (see Supplementary Fig. 10). The cytokines IFN- $\gamma$, TNF- $\alpha$, granzyme B and the membrane protein CD107a are phenotypic markers whose combinatorial expression by lymphocytes is associated with cytotoxic function in multiple contexts, including clinically effective anti-tumor responses ${ }^{13}$. A significant fraction of Immuno-STAT- and peptide-expanded AgS CD8 T cells expressed IFN- $\gamma$, TNF- $\alpha$, granzyme B and CD107a in response to cognate peptide challenge (Fig. If and see Supplementary Fig. 11). Responses to irrelevant peptide were negligible for IFN- $\gamma$, TNF- $\alpha$ and CD107a (see Supplementary Fig. 12). Responses to irrelevant peptide for granzyme B were above zero but generally below responses to cognate peptide for all concentrations measured (see Supplementary Fig. 12). Importantly, ImmunoSTAT-expanded AgS CD8 T cells expressed multiple of these phenotypic markers simultaneously, similar to peptide-expanded AgS CD8 T cells and consistent with differentiation of a potent Teff population (Fig. 1f and see Supplementary Fig. 12). Dose responses were also similar for Immuno-STAT- and peptide-expanded AgS CD8 T cells expressing IFN- $\gamma$, TNF- $\alpha$, granzyme B and CD107a (see Supplementary Fig. 12). Consistent with robust Teff repertoires, tetramer-sorted single cells from peptide or Immuno-STAT expansions showed similar TCR $\alpha \beta$ oligoclonal frequency distributions and sequence-identity was observed between a subset of IST- and peptide-expanded CMV-, but not MART-reactive clones, which were predominantly low clonality (Fig. 1g). 
a

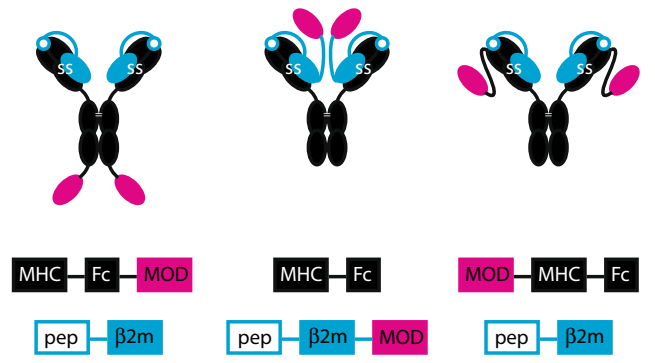

b

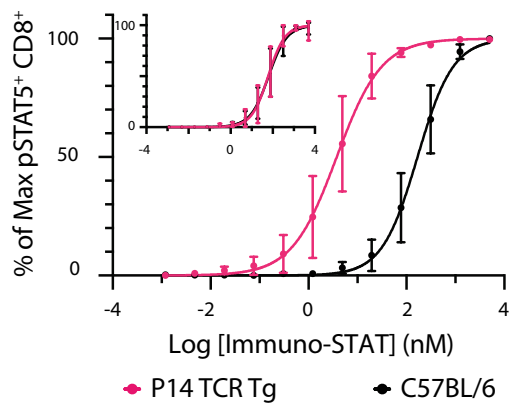

e
C

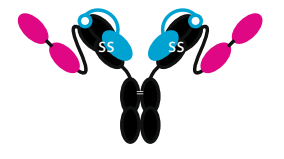

L-2.FH- $-\mathrm{IL}-\mathrm{FH}-\mathrm{HLA}-\mathrm{FC}$

pep $-\beta 2 m$ d

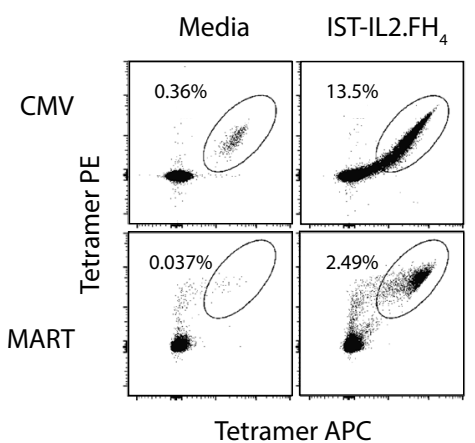

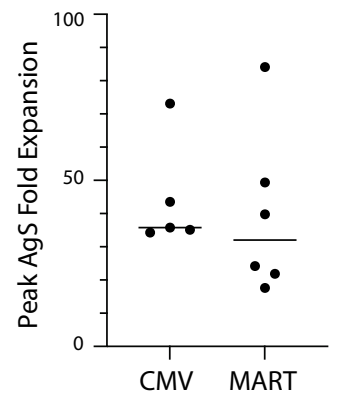

f

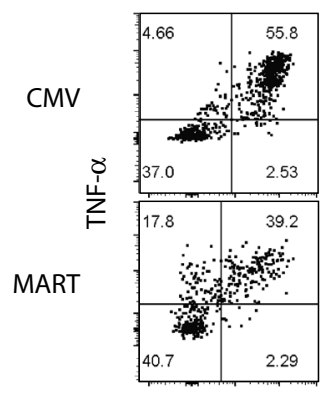

IFN- $\gamma$

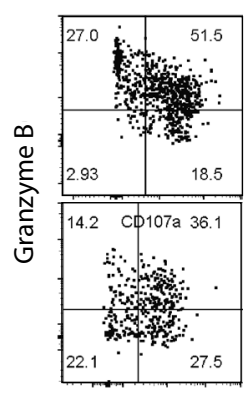

CD107a

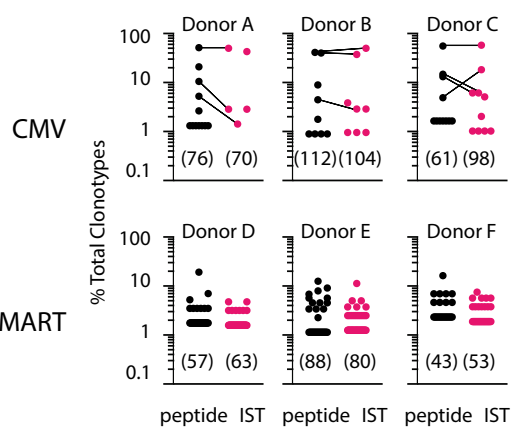

Figure 1. Immuno-STAT design, optimization and activity. (a) Immuno-STAT frameworks comprising peptide (pep) epitope, $\beta 2 \mathrm{~m}$, MHC heavy chain, $\mathrm{Fc}$, and co-modulatory domain (MOD) in different relative positions, covalently linked by engineered (ss) and native (=) disulfide bonds. (b) In vitro pSTAT5 activity of

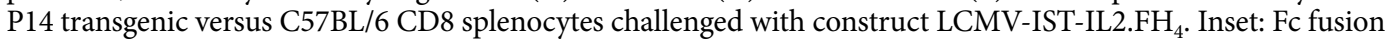
bearing four IL2.FH but without pMHC. \%pSTAT5 ${ }^{+}$responses within each independent dose response titration were normalized as: normalized response $=($ sample - minimum $) /($ maximum - minimum $)$. Data represent mean \pm SD of duplicate samples from three independent experiments. (c) Humanized Immuno-STAT framework comprising two copies of pHLA, four copies of IL2.FH and human IgG1 Fc. (d) Representative dual-tetramer plots of human PBMC stimulated in vitro for ten days with media, CMV-IST-IL2.FH , $_{\text {, }}$ MART-IST-IL2.FH . $_{4}$ (e) Peak fold expansion of AgS T cells from human PBMC incubated with CMV-IST-IL2. $\mathrm{FH}_{4}(\mathrm{n}=5$ donors) and IST-MART-IL2. $\mathrm{FH}_{4}$ ( $\mathrm{n}=6$ donors), from 1 to 4 independent expansions per donor. (f) Representative IFN- $\gamma$, TNF- $\alpha$, CD107a, and granzyme B staining following cognate peptide challenge of Immuno-STAT-expanded CMV- or MART-specific T cells. (g) Paired TCRa $\beta$ sequence frequencies from individual CMV- or MARTspecific CD8 T cells expanded with cognate Immuno-STAT or peptide. Lines connect Immuno-STAT- and peptide-expanded clones having TCR $\beta \beta$ sequence identity. Three donors per specificity. Total clones surveyed in parentheses. 
a

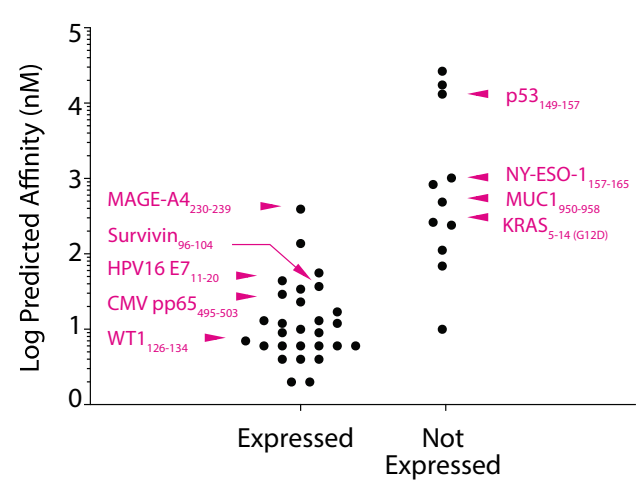

b

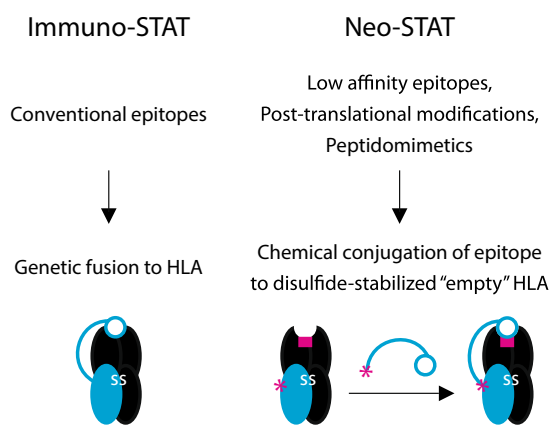

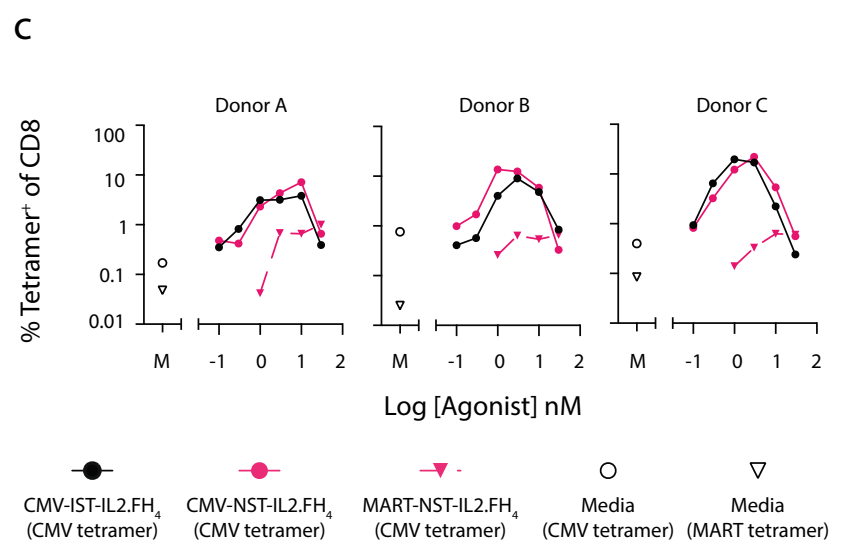

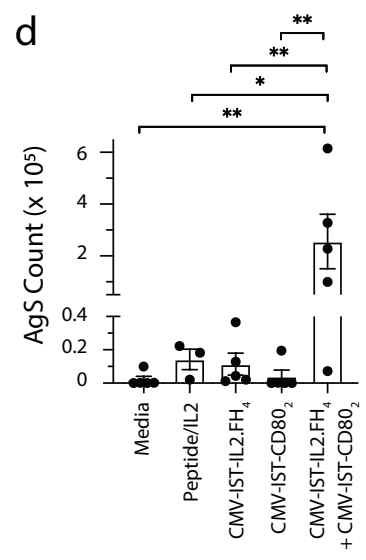

Figure 2. Immuno-STAT and Neo-STAT epitope and co-modulatory flexibility. (a) NetMHC-predicted peptide binding affinity to HLA-A ${ }^{\star} 0201$ versus expressibility on the Immuno-STAT framework, highlighting select epitopes. Data from two independent expression trials with minimum expressibility criteria of $10 \mathrm{mg} / \mathrm{L}$ and $50 \%$ species of interest by SDS-PAGE. (b) Differential application of Immuno-STAT and Neo-STAT frameworks by epitope type. (c) Dual-tetramer frequency of CMV-specific human PBMC T cells following ten day culture with CMV Neo-STAT (CMV-NST-IL2.FH ${ }_{4}$ ), MART-Neo-STAT (MART-NST-IL2.FH ${ }_{4}$ ), CMV-IST-IL2.FH ${ }_{4}$, or media (M). Data are single measurements at each concentration for three donors. (d) CMV-specific human PBMC T cell counts following ten day culture of CMV-IST-IL2. $\mathrm{FH}_{4}$-responsive donors with CMV-IST-IL2.FH 4 and/or CMV-IST-CD $80_{2}$ versus peptide and media controls. Data represent single measurements per condition from 3 to 5 donors. Statistical significance ${ }^{\star}(P \leq 0.05)$ or ${ }^{\star *}(P \leq 0.01)$ assessed by one-tailed paired ratio T test.

The modular Immuno-STAT framework is compatible with diverse co-modulators, peptides and HLA alleles. For example, IL-2, CD80, CD86 and CD137L represent potent costimulatory ligands for CD8 T cells, variants of which can be integrated into the Immuno-STAT framework (see Supplementary Fig. 13). Furthermore, beyond HLA-A ${ }^{\star} 0201$-associated peptides, $\mathrm{AFP}_{403-411} / \mathrm{HLA}-\mathrm{A}^{\star} 1101$ and HBV Protein $\mathrm{P}_{109-118} / \mathrm{HLA}-\mathrm{A}^{\star} 2402$, well characterized cancer and infectious disease antigens, respectively, express well on the Immuno-STAT framework without the comodulator (i.e. pHLA only, see Supplementary Fig. 13). In our hands expression of the Immuno-STAT pHLA is highly predictive of expression of Immuno-STAT pHLA-comodulator combinations such as Immuno-STAT-IL2. $\mathrm{FH}_{4}$. However, epitopes with weak binding to HLA are generally more challenging to express, including clinically significant cancer epitopes such as KRAS G12D (Fig. 2a and see Supplementary Table 3). We therefore developed Neo-STAT, which uses site-specific chemical conjugation of peptides to a stably engineered "empty" HLA, to enable presentation of low-affinity peptides and moieties not accessible via genetic fusion (Fig. 2b). CMV pp65 ${ }_{495-503}$ and $\mathrm{MART}_{26-35}$, model epitopes previously explored on the Immuno-STAT framework, were successfully conjugated to empty HLA-A*0201 on the Neo-STAT framework bearing IL2.FH $\mathrm{FH}_{4}$ (i.e. NST-IL2.FH $\mathrm{FH}_{4}$, see Supplementary Fig. 14). CMV-NST-IL2. $\mathrm{FH}_{4}$ selectively expanded CMV-specific T cells with similar potency to CMV-IST-IL2. $\mathrm{FH}_{4}$ (Fig. 2c). Next, as an initial exploration of co-modulator flexibility and the potential of combinatorial co-modulation, we assessed the ability of CMV-IST bearing the CD80 ectodomain (CMV-IST-CD80 2 ) to induce antigen-specific proliferation in vitro, both as a single agent and when combined with CMV-IST-IL2. $\mathrm{FH}_{4}$. Whereas CMV-IST-CD80 2 alone did not induce AgS proliferation above background levels, CMV-IST-CD80 2 demonstrates synergy with CMV-IST-IL2. $\mathrm{FH}_{4}$ in expanding CMV-specific $\mathrm{T}$ cells in vitro and at levels superior to peptide stimulation (Fig. 2d). 


\section{Discussion}

We have described two modular immunotherapeutics platforms, Immuno-STAT and Neo-STAT, for the copresentation of defined peptide-HLA (pHLA) and co-modulatory ligands to AgS T cells, and engineered an affinity-attenuated variant of IL-2, IL2.FH, for use thereon. IL2.FH-bearing constructs, including an HPV-specific clinical candidate, CUE-101 (NCT03978689), are associated with 110-fold and 3.1-fold decreases in binding to IL2R $\alpha$ and IL2R $\beta$, respectively, with minimal Treg and non-AgS T cell responses in vitro and in vivo ${ }^{14}$. While

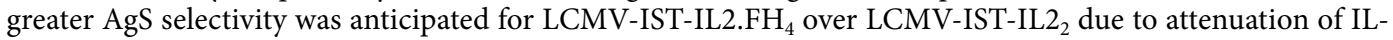
2:IL-2R versus pHLA:TCR binding, this trend was not statistically significant. Potentially more selective variants (e.g. F42A D20K) affecting critical contacts with IL-2R $\beta$ were associated with low peak activities, suggesting trade-offs between selectivity and $\mathrm{Rmax}^{15,16}$. Integration of Immuno-STAT signals through the TCR and IL-2R and/or more IL-2R distal events such as cytokine release may reflect greater selectivity than pSTAT5 induction. Proliferation showed greater selectivity versus pSTAT5 induction for AgS CD8 T cells in response to IST-IL2. $\mathrm{FH}_{4}$, extending similar results obtained with CUE-101. In addition, through modestly attenuated IL-2R $\beta$ binding, H16A may enable strong, but submaximal IL-2 signals, thereby limiting burnout/terminal differentiation while driving proliferation, effector function and memory $\mathrm{T}$ cell generation ${ }^{16,17}$.

AgS cytotoxic CD8 T cell responses have previously been achieved via heterologous expression of membrane pMHC complexes, for example, by DNA vaccination ${ }^{18}$. Alternatively, nanoparticle display of pMHC can stimulate or inhibit AgS T cell responses depending on the presence and nature of costimulatory and co-inhibitory ligands ${ }^{19,20}$. In contrast, soluble pMHC complexes, including streptavidin-based tetramers, largely suppress $\mathrm{T}$ cell responses ${ }^{21,22}$. To our knowledge, Immuno-STAT and Neo-STAT molecules are the first embodiments of soluble pMHC-comodulator signaling complexes directed to AgS T cells in cis.

Immuno-STAT and Neo-STAT access diverse HLA alleles and epitopes in compact architectures which may benefit clinical manufacturability and biodistribution ${ }^{23}$. HLA-A ${ }^{\star} 0201$, HLA-A ${ }^{\star} 1101$ and HLA-A ${ }^{\star} 2402$, surveyed here in the context of Immuno-STAT, are collectively expressed by over half the global population and Neo-STAT extends potential pHLAs to include post-translational modifications and low affinity peptides ${ }^{24}$. In preclinical murine studies, CUE-101 and mCUE-101 elicit AgS T cell responses from both naïve and antigen-experienced precursors, with responses to mCUE-101 detectable in blood, spleen, and tumors, suggesting the potential to both invigorate $\mathrm{T}$ cell responses within tumors and initiate responses outside of immunosuppressive tumor microenvironments, which are relatively resistant to exhaustion ${ }^{14,25}$. Compelling applications of these platforms include enhancing $\mathrm{T}$ cell responses against essential antigens in cancer or infectious disease, especially where relevant AgS T cells are limiting or dysfunctional, and more efficiently activating or maintaining ACT cells ex/in vivo. In addition, conjugating clinical grade peptides and Neo-STAT precursors enables a rapid and parallelizable manufacturing paradigm for immunotherapeutics capable of addressing neoantigens, multi-epitope combinations to address tumor heterogeneity and mitigate tumor escape and rapid responses to emerging pandemics.

As shown here, distinct Immuno-STAT co-modulators can synergize for superior AgS T cell activation in vitro. Moreover, unlike current vaccines, Immuno-STATs and Neo-STATs may bypass APC in vivo to drive therapeutic $\mathrm{T}$ cell responses. The same core architectures bearing appropriate comodulators may also be used to engage other costimulatory pathways, such as CD137 and CD70 26,27. Likewise, Immuno-STATs and NeoSTATs may be used to engage or block inhibitory pathways such as PD-1 and CTLA-4 for AgS activation or downregulation, respectively, while limiting toxicity ${ }^{7}$. Whereas the present work has focused on classical HLA class I restricted T cells, alternative versions of these scaffolds may, in principle, be used to engage any AgS T cell, including T cells specific for non-classical HLA class I ligands as well as HLA class II-restricted ligands.

\footnotetext{
Materials and methods Design, manufacturing, purification, and characterization of Immuno-STAT and Neo-STAT proteins. Immuno-STAT proteins bearing $\mathrm{H}-2 \mathrm{D}^{\mathrm{b}}$ were genetically fused to an effector-attenuated murine $\operatorname{IgG} 2 \mathrm{a}^{28}$. Immuno-STAT and Neo-STAT proteins bearing human HLAs were fused to an effector-attenuated human IgG1 and linked to CMV pp65 ${ }_{495-503}$ or MART1 $1_{26-35}$, immunodominant epitopes in the context of human cytomegalovirus infection or malignant melanoma ${ }^{29-31}$. Domains within the Immuno-STAT and Neo-STAT frameworks are linked via $\mathrm{G}_{4} \mathrm{~S}$ linkers. Cysteine substitutions $\mathrm{R} 12 \mathrm{C}$ of $\beta 2 \mathrm{~m}$ and A236C of HLA-A*0201 result in a stabilizing disulfide bond between these polypeptides. Neo-STAT also contains a disulfide bond comprising cysteine substitutions at Y84C and A139C of HLA- $A^{\star} 0201$, which stabilizes the "empty" (i.e. epitope-less) NeoSTAT precursor prior to conjugation with the peptide epitope of interest ${ }^{32}$. An engineered cysteine, E44C, within $\beta 2 \mathrm{~m}$ serves as the attachment site for maleimide conjugated peptides.

Immuno-STAT and Neo-STAT proteins were expressed by transient transfection in Expi-CHO cells (ThermoFisher). Proteins were purified from the conditioned media using a two-step method of ProteinA capture with MabSelect SuRe (GE) followed by size exclusion chromatography. For SDS-PAGE analysis, proteins were boiled in SDS sample buffer with or without reducing agent for 5 min before loading $2 \mu \mathrm{g}$ per gel lane.

Empty Neo-STAT precursor proteins were linked to maleimide-conjugaged peptides using standard maleimide chemistry. Briefly, empty Neo-STAT precursors were exchanged into and partially reduced with a TCEPbased reducing buffer before two rounds of conjugation with a 20 fold molar excess of peptide-maleimide in the absence of TCEP. Conjugated Neo-STAT proteins were washed at low $\mathrm{pH}$ to remove excess unconjugated peptides in solution before purification by size exclusion and mass confirmation by electrospray ionization time of flight mass spectrometry (ESI-TOF MS).
}

Animals studies. Spleens were collected from C57BL/6J and P14 T cell receptor (specific for LCMV $\mathrm{gp}_{33-41} / \mathrm{H}-2 \mathrm{D}^{\mathrm{b}}$ ) transgenic mice (Jackson Labs) at least six weeks of age following euthanasia by $\mathrm{CO}_{2}$ inhalation and confirmation of euthanasia by cervical dislocation. All studies requiring animal tissues were approved by the 
Institutional Animal Care and Use Committee for SmartLabs (Cambridge, MA) and were performed in compliance with federal guidelines and in accordance with the ARRIVE guidelines 2.0.

pSTAT5 assay and phosphoflow analysis. Spleens were harvested from C57BL/6J and P14 T cell receptor (specific for LCMV gp $33-41$ /H-2D ${ }^{b}$ ) transgenic mice (Jackson Labs). Splenocytes from 10 to 15 mice (depending on the number of conditions tested) per strain were pooled and CD8 T cells were isolated using Dynabeads Untouched Mouse CD8 kit (ThermoFisher). CD8 splenocytes were resuspended in RPMI culture media (ATCC) supplemented with $10 \%$ fetal bovine serum (Hyclone). $1 \times 10^{5}$ C57BL/6J CD8 splenocytes or P14 TCR Tg CD8 splenocytes were seeded in 96 -well U-bottom plates and stimulated with Immuno-STAT at a given concentration per well for $20 \mathrm{~min}$ at $37^{\circ} \mathrm{C}$ (initial Immuno-STAT-IL2 framework variant screen) or $5 \mathrm{~min}$ at $37^{\circ} \mathrm{C}$ (follow up studies) in a final volume of $100 \mu \mathrm{l}$. For the initial Immuno-STAT framework variant screen, mouse splenic CD8 T cells were incubated with Immuno-STAT proteins at 0.01, 0.1, 1, 10, 100, 250, 500 and $1000 \mathrm{nM}$. For

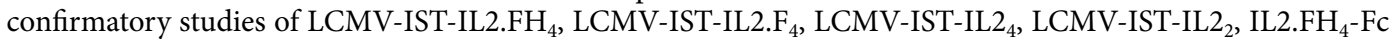
and rhIL-2 (Peprotech), mouse splenic CD8 T cells were incubated with test articles at 0.00119, 0.00477, 0.0191, $0.0763,0.305,1.22,4.88,19.5,78.1,313,1250$, and $5000 \mathrm{nM}$. Cells were immediately fixed with IC fixation buffer (ThermoFisher), permeabilized with $100 \%$ methanol, and stained with a $1 / 10$ dilution of anti-pY694 STAT5 antibody (BD Biosciences) for $30 \mathrm{~min}$ at room temperature, followed by flow cytometry on an iQue Screener (Intellicyt) for initial IST variant screen or an Attune NxT cytometer (Invitrogen) for follow up studies. The percent of positively stained cells was determined using FlowJo software (TreeStar). Non-linear curve fits and EC50 determinations from pSTAT5 dose response data were performed using Prism analysis software (Graphpad).

Human T cell expansion, tetramer staining and flow cytometry analysis. Human healthy donor peripheral blood mononuclear cells (PBMC) were obtained as frozen stocks (Astarte Biologics) or isolated from leukopaks (HemaCare); washed and resuspended in ImmunoCult-XF Cell Expansion Media (Stemcell Technologies). $1 \times 10^{7} \mathrm{PBMC}$ were seeded in a 6 well plate with specific Immuno-STAT or Neo-STAT at a given concentration or media control in a total volume of $4 \mathrm{ml}$. Cells were incubated with $0,0.1,1,3,10,30$, or $100 \mathrm{nM}$ CMVIST-IL2. $\mathrm{FH}_{4}$ or MART-IST-IL2. $\mathrm{FH}_{4}$, with each concentration evaluated in 1-4 independent expansion trials. $0.3 \mathrm{nM}$ Immuno-STAT was also evaluated for CMV-IST-IL2. $\mathrm{FH}_{4}$. For Neo-STAT expansion studies, PBMC were incubated with CMV-IST-FH ${ }_{4}$ or CMV-NST-FH $\mathrm{CH}_{4}$ at $0,0.1,0.3,1,3,10$ and $30 \mathrm{nM}$ and PBMC were incubated with control MART-NST-IL2. $\mathrm{FH}_{4}$ at $0,1,3,10$ and $30 \mathrm{nM}$. For experiments examining the combined activity of CMV-IST-IL2. $\mathrm{FH}_{4}$ and CMV-IST-CD80 2 , cells were incubated with CMV-IST-IL2.FH ${ }_{4}$ and CMV-IST-CD80 2 at 1 and $100 \mathrm{nM}$, respectively, or with media alone or with $5 \mu \mathrm{g} / \mathrm{ml} \mathrm{CMV} \mathrm{pp65_{495-503 }}$ (NLVPMVATV) peptide plus $50 \mathrm{IU} / \mathrm{ml} \mathrm{IL}-2$. Immuno-STAT or peptide expansion cultures were maintained in a $37^{\circ} \mathrm{C} \mathrm{CO}_{2}$ incubator with replacement of half the culture media at day 5 and day 7. Cells were harvested on day 10, washed, resuspended in FACS buffer on ice and stained for viability using Fixable Viability Stain 780 (BD Biosciences) before staining with relevant tetramers (MBL International, MA) on ice for $30 \mathrm{~min}$. Tetramer-stained cells were then washed and stained on ice with antibodies against CD3 (clone SK7, BioLegend), CD14 (clone M5E2, BioLegend), CD19 (clone HIB19, BioLegend), CD56 (clone HCD56, BioLegend), CD4 (clone SK3, BioLegend) and CD8 (clone SK1, BD Biosciences) for $30 \mathrm{~min}$. Tetramer flow cytometric data was acquired using the Attune NxT cytometer (ThermoFisher) and analyzed using FlowJo software (Tree Star). Peak fold expansion per donor per trial was calculated as the maximum tetramer-positive frequency observed for PBMC expanded with specific IST-IL2. $\mathrm{FH}_{4}$ over tetramer-positive frequency for media-incubated PBMC. Peak fold expansion data was derived from between one and three expansion trials per donor, with mean peak fold expansion values used for donors with multiple expansion trials.

Intracellular cytokine and phenotypic staining of in vitro-expanded human CD8 T cells. A total of 2 to $4 \times 10^{6}$ human PBMCs expanded with specific Immuno-STAT or peptide were pretreated with brefeldin A (BFA) and monensin (ThermoFisher), plated in a 24-well plate, and stimulated at a 1:1 ratio with

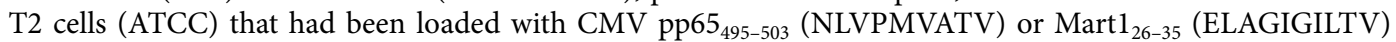

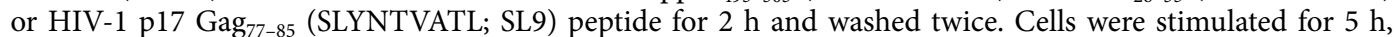
washed, stained with Fixed Viability Stain 780 (BD Biosciences), antibodies against CD3 (clone SK7, BioLegend), CD8 (clone SK1, BD Biosciences) and CD107a (clone H4A3, BD Biosciences), and fixed using IC fixation buffer (ThermoFisher). Cells were next washed in permeabilization buffer (eBioscience), stained with antibodies against TNF- $\alpha$ (clone MAb11, BD Biosciences), IFN- $\gamma$ (clone 4S.B3, BioLegend), and granzyme B (clone GB11, $\mathrm{BD}$ Biosciences) for $30 \mathrm{~min}$ at room temperature, washed, and analyzed. For representative FACS plots and pairwise marker quantitation, PBMC were stimulated as described above using T2 cells loaded with $100 \mathrm{nM}$ peptide. For peptide titration studies, PBMC were stimulated as described above using T2 cells loaded with $1 \times 10^{-13}$, $1 \times 10^{-12}, 1 \times 10^{-11}, 1 \times 10^{-10}, 1 \times 10^{-9}, 1 \times 10^{-8}, 1 \times 10^{-7}, 1 \times 10^{-6}$, or $1 \times 10^{-5} \mathrm{~g} / \mathrm{ml}$ peptide.

TCR sequencing. PBMCs from healthy donors were expanded in vitro with CMV pp65 ${ }_{495-503}$ or Mart $_{26-35}$ peptide plus IL-2, or with $100 \mathrm{nM}$ specific Immuno-STAT in 10-day cultures. Expanded cells were harvested, tetramer stained, and CMV- or MART1-specific CD8 ${ }^{+} \mathrm{T}$ cells were single cell sorted (Sony SH800) and the $\alpha$ and $\beta$ TCR chains were coamplified (iRepertoire). TCR library sequencing used an Illumina MiSeq v2 Nano Kit. Data for each individual well were demultiplexed, mapped, and analyzed using the iRmap VDJ pipeline and the iPair Analyzer. 
Received: 11 June 2021; Accepted: 6 September 2021

Published online: 28 September 2021

\section{References}

1. Yang, J. C. \& Rosenberg, S. A. Adoptive T-cell therapy for cancer. Adv. Immunol. 130, 279-294 (2016).

2. Segal, N. H. et al. Results from an integrated safety analysis of urelumab, an agonist anti-CD137 monoclonal antibody. Clin. Cancer Res. 23, 1929-1936 (2017).

3. Klebanoff, C. A., Acquavella, N., Yu, Z. \& Restifo, N. P. Therapeutic cancer vaccines: Are we there yet. Immunol. Rev. 239, 27-44 (2011).

4. Dutcher, J. P. et al. High dose interleukin-2 (Aldesleukin)—Expert consensus on best management practices-2014. J. Immunother. Cancer 2, 26 (2014).

5. Shang, B., Liu, Y., Jiang, S. J. \& Liu, Y. Prognostic value of tumor-infiltrating FoxP3+ regulatory T cells in cancers: A systematic review and meta-analysis. Sci. Rep. 5, 15179 (2015).

6. Marchingo, J. M. et al. T cell signalling. Antigen affinity, costimulation, and cytokine inputs sum linearly to amplify T cell expansion. Science 346, 1123-1127 (2014).

7. Larkin, J. et al. Combined nivolumab and ipilimumab or monotherapy in untreated melanoma. N. Engl. J. Med. 373, 23-34 (2015).

8. Wculek, S. K. et al. Dendritic cells in cancer immunology and immunotherapy. Nat. Rev. Immunol. 20, 7-24 (2020).

9. Bakdash, G., Sittig, S. P., van Dijk, T., Figdor, C. G. \& de Vries, I. J. The nature of activatory and tolerogenic dendritic cell-derived signal II. Front. Immunol. 4, 53 (2013).

10. Stone, J. D. \& Stern, L. J. CD8 T cells, like CD4 T cells, are triggered by multivalent engagement of TCRs by MHC-peptide ligands but not by monovalent engagement. J. Immunol. 176, 1498-1505 (2006).

11. Krieg, C., Létourneau, S., Pantaleo, G. \& Boyman, O. Improved IL-2 immunotherapy by selective stimulation of IL-2 receptors on lymphocytes and endothelial cells. Proc. Natl. Acad. Sci. USA 107, 11906-11911 (2010).

12. Ghelani, A. et al. Defining the threshold IL-2 signal required for induction of selective Treg cell responses using engineered IL-2 muteins. Front. Immunol. 11, 1106 (2020).

13 Ramesh, P., Shivde, R., Jaishankar, D., Saleiro, D. \& Le Poole, I. C. A palette of cytokines to measure anti-tumor efficacy of T cellbased therapeutics. Cancers 13, 821 (2021).

14. Quayle, S. N. et al. CUE-101, a novel E7-pHLA-IL2-Fc fusion protein, enhances tumor antigen-specific T-cell activation for the treatment of HPV16-driven malignancies. Clin. Cancer Res. 26, 1953-1964 (2020).

15. Wang, X., Rickert, M. \& Garcia, K. C. Structure of the quaternary complex of interleukin-2 with its alpha, beta, and gammac receptors. Science 310, 1159-1163 (2005).

16. Mitra, S. et al. Interleukin-2 activity can be fine tuned with engineered receptor signaling clamps. Immunity 42, 826-838 (2015).

17. Kalia, V. et al. Prolonged interleukin-2Ralpha expression on virus-specific CD8+ T cells favors terminal-effector differentiation in vivo. Immunity 32, 91-103 (2010).

18. Kim, S. et al. Single-chain HLA-A2 MHC trimers that incorporate an immundominant peptide elicit protective T cell immunity against lethal West Nile virus infection. J. Immunol. 184, 4423-4430 (2010).

19. Perica, K., Kosmides, A. K. \& Schneck, J. P. Linking form to function: Biophysical aspects of artificial antigen presenting cell design. Biochim. Biophys. Acta 1853, 781-790 (2015).

20. Serra, P. \& Santamaria, P. Peptide-MHC-based nanomedicines for the treatment of autoimmunity: Engineering, mechanisms, and diseases. Front. Immunol. 11, 621774 (2020).

21. Cebecauer, M. et al. Soluble MHC-peptide complexes induce rapid death of CD8+ CTL. J. Immunol. 174, 6809-6819 (2005).

22. Angelov, G. S. et al. Soluble MHC-peptide complexes containing long rigid linkers abolish CTL-mediated cytotoxicity. J. Immunol. 176, 3356-3365 (2006)

23. Woodham, A. W. et al. In vivo detection of antigen-specific $\mathrm{CD} 8^{+} \mathrm{T}$ cells by immuno-positron emission tomography. Nat. Methods 17, 1025-1032 (2020).

24. González-Galarza, F. F. et al. Allele frequency net 2015 update: New features for HLA epitopes, KIR and disease and HLA adverse drug reaction associations. Nucleic Acids Res. 43, D784-D788 (2015).

25. Nguyen, H. H. et al. Naïve CD8(+) T cell derived tumor-specific cytotoxic effectors as a potential remedy for overcoming TGF- $\beta$ immunosuppression in the tumor microenvironment. Sci. Rep. 6, 28208 (2016).

26. Habib-Agahi, M., Phan, T. T. \& Searle, P. F. Co-stimulation with 4-1BB ligand allows extended T-cell proliferation, synergizes with CD80/CD86 and can reactivate anergic T cells. Int. Immunol. 19, 1383-1394 (2007).

27. Starzer, A. M. \& Berghoff, A. S. New emerging targets in cancer immunotherapy: CD27 (TNFRSF7). ESMO Open 4, e000629 (2020).

28. Steurer, W. et al. Ex vivo coating of islet cell allografts with murine CTLA4/Fc promotes graft tolerance. J. Immunol. 155, 1165-1174 (1995).

29. Wills, M. R. et al. The human cytotoxic T-lymphocyte (CTL) response to cytomegalovirus is dominated by structural protein pp65: Frequency, specificity, and T-cell receptor usage of pp65-specific CTL. J Virol 70, 7569-7579 (1996).

30. Romero, P. et al. Antigenicity and immunogenicity of Melan-A/MART-1 derived peptides as targets for tumor reactive CTL in human melanoma. Immunol. Rev. 188, 81-96 (2002).

31. Hezareh, M., Hessell, A. J., Jensen, R. C., van de Winkel, J. G. \& Parren, P. W. Effector function activities of a panel of mutants of a broadly neutralizing antibody against human immunodeficiency virus type 1. J. Virol. 75, 12161-12168 (2001)

32. Hein, Z. et al. Peptide-independent stabilization of MHC class I molecules breaches cellular quality control. J. Cell Sci. 127, 2885-2897 (2014)

\section{Author contributions}

S.C.A., R.S. and R.C. conceived the IST technology and R.S., R.C., S.L and J.R. conceived the NST technology; R.S., R.C. and S.C. designed experiments, analysed data and made figures; R.S. and R.C wrote the manuscript; J.R., S.L., S.Q., P.K. and A.S. suggested experiments and interpreted data; J.R., S.L., J.S., P.R., W.B. and A.V. designed, manufactured, purified, and characterized IST and NST reagents; Z.M. and D.T. performed pSTAT5 and $T$ cell expansion assays and provided flow cytometric analysis; D.T. performed intracellular cytokine and phenotypic staining; E.S. performed TCR sequencing. All authors reviewed the manuscript.

\section{Competing interests}

Initial work underlying the Immuno-STAT ${ }^{\mathrm{ma}}$ technology was performed in the laboratory of S.C.A., of which S.C.A., R.S. and R.C. are co-founders and are co-inventors on SynTac Polypeptides and Uses Thereof (PCT/2015/195531 and corresponding patents and applications), which are licensed to Cue Biopharma, Inc. and relate to the Immuno-STAT platform. R.S., R.C., S.L and J.R. are co-inventors of other patents and patent 
applications that are owned by Cue Biopharma, Inc. and relate to the Immuno-STAT and Neo-STAT ${ }^{\mathrm{mm}}$ platforms. S.C.A., R.S., A.S and R.C. are stockholders in Cue Biopharma, Inc.; R.S., Z.M., J.S., P.R., W.B., S.Q., J.F. and A.S. are employee's of Cue Biopharma, Inc. All other co-authors declare no competing interest.

\section{Additional information}

Supplementary Information The online version contains supplementary material available at https://doi.org/ 10.1038/s41598-021-98716-Z.

Correspondence and requests for materials should be addressed to R.D.S.

Reprints and permissions information is available at www.nature.com/reprints.

Publisher's note Springer Nature remains neutral with regard to jurisdictional claims in published maps and institutional affiliations.

Open Access This article is licensed under a Creative Commons Attribution 4.0 International format, as long as you give appropriate credit to the original author(s) and the source, provide a link to the Creative Commons licence, and indicate if changes were made. The images or other third party material in this article are included in the article's Creative Commons licence, unless indicated otherwise in a credit line to the material. If material is not included in the article's Creative Commons licence and your intended use is not permitted by statutory regulation or exceeds the permitted use, you will need to obtain permission directly from the copyright holder. To view a copy of this licence, visit http://creativecommons.org/licenses/by/4.0/.

(C) The Author(s) 2021 